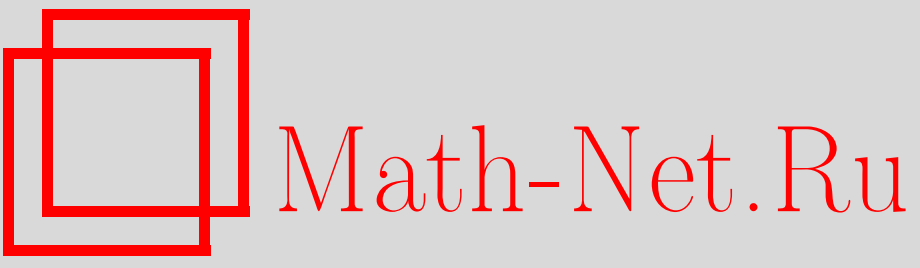

Н. Г. Мощевитин, Об одной теореме Пуанкаре, УМН, 1998, том 53, выпуск 1, 223-224

DOI: https://doi.org/10.4213/rm26

Использование Общероссийского математического портала Math-Net.Ru подразумевает, что вы прочитали и согласны с пользовательским соглашением

http://www . mathnet.ru/rus/agreement

Параметры загрузки:

IP: 35.174 .16 .151

26 апреля 2023 г., 13:50:46 


\section{ОБ ОДНОЙ ТЕОРЕМЕ ПУАНКАРЕ}

\section{Н. Г. МОщЕВитин}

1. Рассматривается компактное многообразие $M, \operatorname{dim} M=d$ (или просто ограниченная область в $\mathbb{R}^{d}$ ) и преобразование $g: M \rightarrow M$, сохраняющее объем. Хорошо известно, что почти всякая траектория $g^{t} x$ возвращается, т.е. имеет место соотношение

$$
\forall \varepsilon>0 \forall T>0 \quad \exists t>T: \rho\left(g^{t} x, x\right)<\varepsilon .
$$

(Здесь и далее $\rho(\cdot, \cdot)$ означает некоторое расстояние на $M$. .) Этот простой результат называется теоремой Пуанкаре о возвращаемости (см. [1]). В настоящей заметке дается несложный (основьвающийся только на лемме Бореля-Кантелли) количественный вариант этой теоремы.

ТЕоремА. Пусть положительнозначная функция $\psi(t)$ вещественного аргумента сколь угодно медленно возрастает $\kappa+\infty$ при $t \rightarrow+\infty$ u $\psi(t) / t^{1 / d}$ монотонно убивает $\kappa 0$ при $t \rightarrow+\infty$. Тогда для почти каждого (в смысле меры Лебега) $x \in M$ найдется последовательность $t_{\nu} \rightarrow+\infty$ такая, что

$$
\rho\left(g^{t_{\nu}} x, x\right)<t_{\nu}^{-1 / d} \psi\left(t_{\nu}\right)
$$

ЗАмечАниЕ 1. Известная теорема из теории алгебраических чисел (см. [2]) утверждает, что для чисел $\alpha_{1}, \ldots, \alpha_{d}, d \geqslant 1$, образующих вместе с единицей базис некоторого вещественного алгебраического поля степени $d+1$, выполнено

$$
\max _{1 \leqslant j \leqslant d} \min _{a_{j} \in \mathbb{Z}}\left|\alpha_{j} t-a_{j}\right| \geqslant C(\alpha) t^{-1 / d} \quad \forall t \in \mathbb{N} .
$$

Таким образом, приведен пример сохранющего объем сдвига $g$ на торе $\mathbb{T}^{d}$, для которого

$$
\rho\left(g^{t} x, x\right)>C t^{-1 / d} \quad \forall t \in \mathbb{N} \quad \forall x \in \mathbb{T}^{d},
$$

и показатель $-1 / d$ при степени $t$ в нашей теореме неулучшаем.

ЗАмечАниЕ 2. Очевидно, имеет место неравенство

$$
\sup _{x \in M} \min _{0 \leqslant t<\tau \leqslant T} \rho\left(g^{\tau} x, g^{t} x\right)<C(M) T^{-1 / d}
$$

с некоторой постоянной $C(M)$, зависящей толњко от $M$, причем sup и $\min$ в этой формуле, вообще говоря, переставить нелњзя.

СлеДСтвиЕ. Пусть на компактном многообразии $M, \operatorname{dim} M=d$, имеется вектор-

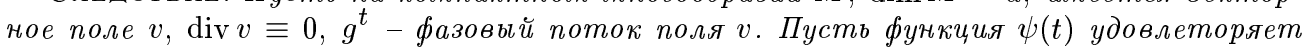
условию нашей теоремы. Тогда для почти каждого (в смысле меры Лебега) $x \in M$ найдется последовательность $t_{\nu} \rightarrow+\infty$ такая, что

$$
\rho\left(g^{t_{\nu}} x, x\right)<t_{\nu}^{-1 / d} \psi\left(t_{\nu}\right)
$$

Работа выполнена при финансовой поддержке Российского фонда фундаментальных исследований (гранты № 96-01-00378 и № 96-15-96072). 
2. Доказательство теоремы. Пусть $\sigma \subset M$ - множество тех точек из $M$, для которых утверждение теоремы не выполнено:

$$
\sigma=\left\{x \in M: \exists k_{0} \in \mathbb{N} \forall k \geqslant k_{0}, k \in \mathbb{N} \min _{k_{0} \leqslant q \leqslant k, q \in \mathbb{N}} \rho\left(g^{q} x, x\right) \geqslant k^{-1 / d} \psi(k)\right\} .
$$

Пусть

$$
\sigma_{k_{0}, k}=\left\{x \in M: \min _{k_{0} \leqslant q \leqslant k, q \in \mathbb{N}} \rho\left(g^{q} x, x\right) \geqslant k^{-1 / d} \psi(k)\right\} .
$$

Ясно, что все $\sigma_{k_{0}, k}$ измеримші,

$$
\begin{aligned}
\sigma_{k_{0}, k} & \subseteq \sigma_{k_{0}^{\prime}, k} \text { при } k_{0}^{\prime} \geqslant k_{0}, \\
\sigma & =\bigcup_{k_{0}=0}^{+\infty} \bigcap_{k=k_{0}}^{+\infty} \sigma_{k_{0}, k} .
\end{aligned}
$$

Пусть $\mathscr{A}_{k}=\{A\}$ - некоторое разбиение $M$ на квадрируемые кусочки $A$, удовлетворяющее свойствам

$$
k^{-1 / d} \psi(k) \ll \operatorname{diam} A \leqslant k^{-1 / d} \psi(k), \quad \mu(A) \asymp k^{-1}(\psi(k))^{d},
$$

Обозначим $U_{k}(x)=\left\{y \in M: \rho(x, y)<k^{-1 / d} \psi(k)\right\}$. Ясно, что для $x \in A$ выполнено $A \subseteq U_{k}(x)$. Рассмотрим множество $\sigma_{k_{0}, k}(A)=\sigma_{k_{0}, k} \cup A$ и оценим его меру. Множества

$$
g^{0} \sigma_{k_{0}, k}(A), g^{k_{0}} \sigma_{k_{0}, k}(A), \ldots, g^{\left[k / k_{0}\right] k_{0}} \sigma_{k_{0}, k}(A)
$$

попарно не пересекаются (это следует из определения $\left.\sigma_{k_{0}, k}(A)\right)$, а $g^{t}$ сохраняет объем (как отмечалось в начале заметки). Из ограниченности $M$ следует, что $\mu\left(\sigma_{k_{0}, k}(A)\right) \ll k^{-1} k_{0}$. Далее,

$$
\mu\left(\sigma_{k_{0}, k}\right) \leqslant \sum_{A \in \mathscr{A}} \mu\left(\sigma_{k_{0}, k}(A)\right) \ll \# \mathscr{A}_{k} k^{-1} k_{0} \ll k_{0}(\psi(k))^{-d}
$$

(в последнем неравенстве учтено (3)).

Выберем теперь возрастающие к бесконечности последовательности натуральных чисел $\mu_{\nu}, \lambda_{\nu} \rightarrow+\infty, \nu \rightarrow+\infty$ так, чтобы ряд

$$
\sum_{\nu=0}^{+\infty} \mu_{\nu}\left(\psi\left(\lambda_{\nu}\right)\right)^{-d}
$$

сходился. Из (1), (2) следует, что

$$
\sigma \subseteq \bigcup_{p=w}^{+\infty} \sigma_{\mu_{p}, \lambda_{p}} \quad \forall w \in \mathbb{N} .
$$

Посколшку ряд $\sum_{\nu=0}^{+\infty} \mu\left(\sigma_{\mu_{\nu}}, \lambda_{\nu}\right)$ сходится в силу оценки (4) и выбора последовательностей $\mu_{\nu}$, $\lambda_{\nu}$, получаем, что $\mu(\sigma)=0$. Теорема доказана.

\section{СПИСОК ЛИТЕРАТУРЫ}

[1] Пуанкаре А. Новые методы небесной механики // Избранные труды. Т. 2. М.: Наука, 1972. [2] Касселс Дж. Введение в теорию диофантовых приближений: М., 1961. 